\title{
The role of perineal application of prophylactic negative-pressure wound therapy for prevention of wound-related complications after abdomino-perineal resection: a systematic review
}

\author{
Jeremy Meyer ${ }^{1}$ (D) Elin Roos ${ }^{2} \cdot$ Ziad Abbassi $^{1} \cdot$ Christian Toso $^{1} \cdot$ Frédéric Ris ${ }^{1} \cdot$ Nicolas C. Buchs ${ }^{1}$
}

Accepted: 25 August 2020 / Published online: 4 September 2020

(C) The Author(s) 2020

\begin{abstract}
Background Closed perineal wounds often fail to heal by primary intention after abdomino-perineal resection (APR) and are often complicated by surgical site infection (SSI) and/or wound dehiscence. Recent evidence showed encouraging results of prophylactic negative-pressure wound therapy (pNPWT) for prevention of wound-related complications in surgery. Our objective was to gather and discuss the early existing literature regarding the use of pNPWT to prevent wound-related complications on perineal wounds after APR. Methods Medline, Embase, and Web of Science were searched for original publications and congress abstracts reporting the use of pNPWT after APR on closed perineal wounds.

Results Seven publications were included for analysis. Two publications reported significantly lower incidence of SSI in pNPWT patients than in controls with a risk reduction of about 25-30\%. Two other publications described similar incidences of SSI between the two groups of patients but described SSI in pNPWT patients to be less severe. One study reported significantly lower incidence of wound dehiscence in pNPWT patients than in controls.

Conclusion The largest non-randomized studies investigating the effect of pNPWT on the prevention of wound-related complications after APR showed encouraging results in terms of reduction of SSI and wound dehiscence that deserve further investigation and confirmation.
\end{abstract}

Keywords Abdomino-perineal amputation $\cdot$ Abdomino-perineal resection $\cdot$ Colorectal cancer $\cdot$ Rectal cancer $\cdot$ Infection $\cdot$ Perineal infection

\section{Introduction}

Abdomino-perineal resection (APR) of the rectum consists of the ablation of the terminal colon, the rectum, the internal and external sphincters, and the confection of a terminal colostomy, as initially described by Miles [1]. APR is usually indicated for

Electronic supplementary material The online version of this article (https://doi.org/10.1007/s00384-020-03732-6) contains supplementary material, which is available to authorized users.

Jeremy Meyer

jeremy.meyer@hcuge.ch

1 Division of Digestive Surgery, University Hospitals of Geneva, Rue Gabrielle-Perret-Gentil 4, 1211 Genève 14, Switzerland

2 Department of Global Public Health, Karolinska Institute, Stockholm, Sweden advanced adenocarcinomas of the lower third of the rectum (within $5 \mathrm{~cm}$ from the anal verge) and for recurrent squamous cell carcinoma of the rectum or anal margin after chemo-radiotherapy. Improvements to the techniques include neoadjuvant radio-chemotherapy for stages T 3-4 and/or radiologically node-positive adenocarcinomas, synchronous abdominal and perineal approaches, total mesorectum excision (TME, as introduced by Heald [2]), and minimally invasive techniques avoiding laparotomy for the abdominal approach.

Recently, emergence of sphincter-sparing procedures, such as partial and total intersphincteric resections for adenocarcinomas $<1 \mathrm{~cm}$ from the internal sphincter but sparing the external sphincter allowed reducing the indication for APR in favor of anterior resection. However, APR is still performed for rectal adenocarcinomas extending to the external sphincter, for incontinent patients, and for recurrent squamous cell carcinoma. Further, wider resections, such as extralevator 
abdomino-perineal excision (ELAPE [3]) removing the totality of levator ani muscles from their origin associated or not to multivisceral resection, are sometimes required in case of infiltration of levator ani muscles or surrounding organs, although indication for ELAPE is still debatable.

APR results in wide perineal defects. Usually, levator ani muscles are reapproximated using absorbable stiches. If a gap remains, the subcutaneous fat in the ischiorectal space or a synthetic or biological mesh can be used to fill the empty space [4]. In some cases, reconstruction using flaps, such as pedicled vertical rectus abdominis myocutaneous flap, local V-to-Y advancement flap (inferior gluteal artery perforator flap), and pedicled gracilis muscle flaps, is required $[5,6]$.

However, perineal wounds often fail to heal, notably due to preoperative radiotherapy side effects $[7,8]$, resulting in significant morbidity for patients, prolonged hospitalization, and increased costs for the healthcare system [9]. After failure to heal, a conventional negative-pressure wound therapy (NPWT) device (usually V.A.C .) is usually put in place to help healing by secondary intention.

Recently, NPWT preventively applied on closed wounds, also named prophylactic NPWT (pNPWT) or incisional NPWT (iNPWT), was reported to lower the risk of SSI after surgery in various surgical specialties, notably in gastrointestinal surgery [10-12]. Of interest, early publications reported encouraging results after APR, for which surgical wounds are more at risk of complications, but pooled evidence is lacking in that context.

The primary objective of the present systematic review was to gather and discuss the early existing literature regarding the use of pNPWT to prevent wound-related complications, notably SSI, on perineal wounds after APR.

\section{Methods}

This systematic review was performed according to the Preferred Reporting Items for Systematic Reviews and Meta-Analyses (PRISMA) guidelines [13] (Table S1). MEDLINE, Embase, and Web of Science were searched from inception to 8 November 2019 for original studies written in English, Swedish, or French including patients who benefited from perineal application of pNPWT after APR. Search strategy is summarized in Table 1. Case series and conference abstracts were considered. Additional records were identified by manual search of the reference lists of the included publications. Secondary analyses of previously published papers and studies including patients $<18$ years old were excluded. Studies were screened for inclusion by two authors (ER, JM). Discrepancies were solved by a third author (NCB). The systematic review and meta-analysis protocol was registered in the International Prospective Register of Ongoing Systematic Reviews (PROSPERO).

\section{Results}

\section{Inclusion process}

Seventy-seven publications were identified from MEDLINE, 74 from Embase and 14 from Web of Science. One hundred and fifty-seven records were excluded after abstract/title screening, and one after full-text screening, leaving seven publications [14-20] for definitive inclusion (Fig. 1).

Table 1 Literature search strategy

\begin{tabular}{ll}
\hline Database & Search build \\
\hline MEDLINE & (Negative-pressure[Title/Abstract]) OR (Negative pressure[Title/Abstract]) OR (Negative-pressure \\
& therapy[Title/Abstract]) OR (Negative pressure therapy[Title/Abstract]) \\
& OR (Negative-pressure wound therapy[Title/Abstract]) OR (Negative pressure wound therapy[Title/Abstract]) OR \\
& (Prophylactic closed-incision negative-pressure wound therapy[Title/Abstract]) OR (Prophylactic closed-incision \\
& negative pressure wound therapy[Title/Abstract]) OR (NPT[Title/Abstract]) OR (NPWT[Title/Abstract]) OR \\
& (pNPT[Title/Abstract]) OR (pNPWT[Title/Abstract])) \\
& AND \\
& (perineal[Title/Abstract]) OR (perineum[Title/Abstract]) OR (abdominoperineal resection[Title/Abstract]) OR \\
& (abdomino-perineal resection[Title/Abstract]) OR (abdominoperineal excision[Title/Abstract]) OR \\
& (abdomino-perineal excision[Title/Abstract]) OR (APE[Title/Abstract]) OR (APR[Title/Abstract])) \\
& ('negative-pressure therapy':ti,ab,kw OR 'negative pressure therapy':ti,ab,kw OR 'negative-pressure wound \\
therapy':ti,ab,kw OR 'negative pressure wound therapy':ti,ab,kw OR 'NPWT': \\
ti,ab,kw) \\
AND \\
EMBASE & ('perineal':ti,ab,kw OR 'perineum':ti,ab,kw OR 'abdominoperineal resection':ti,ab,kw OR 'abdomino-perineal \\
& resection':ti,ab,kw OR 'abdominoperineal excision':ti,ab,kw OR 'abdomino-perineal excision':ti,ab,kw OR \\
& 'APE':ti,ab,kw OR 'APR':ti,ab,kw) \\
& TI $=($ negative-pressure therapy OR negative pressure therapy OR negative-pressure wound therapy OR negative \\
pressure wound therapy OR NPWT) & 14 \\
ANDTI = (perineal OR perineum OR abdominoperineal resection OR abdomino-perineal resection OR \\
abdominoperineal excision OR abdomino-perineal excision OR APE OR APR) \\
WEB OF
\end{tabular}


Fig. 1 PRISMA flowchart

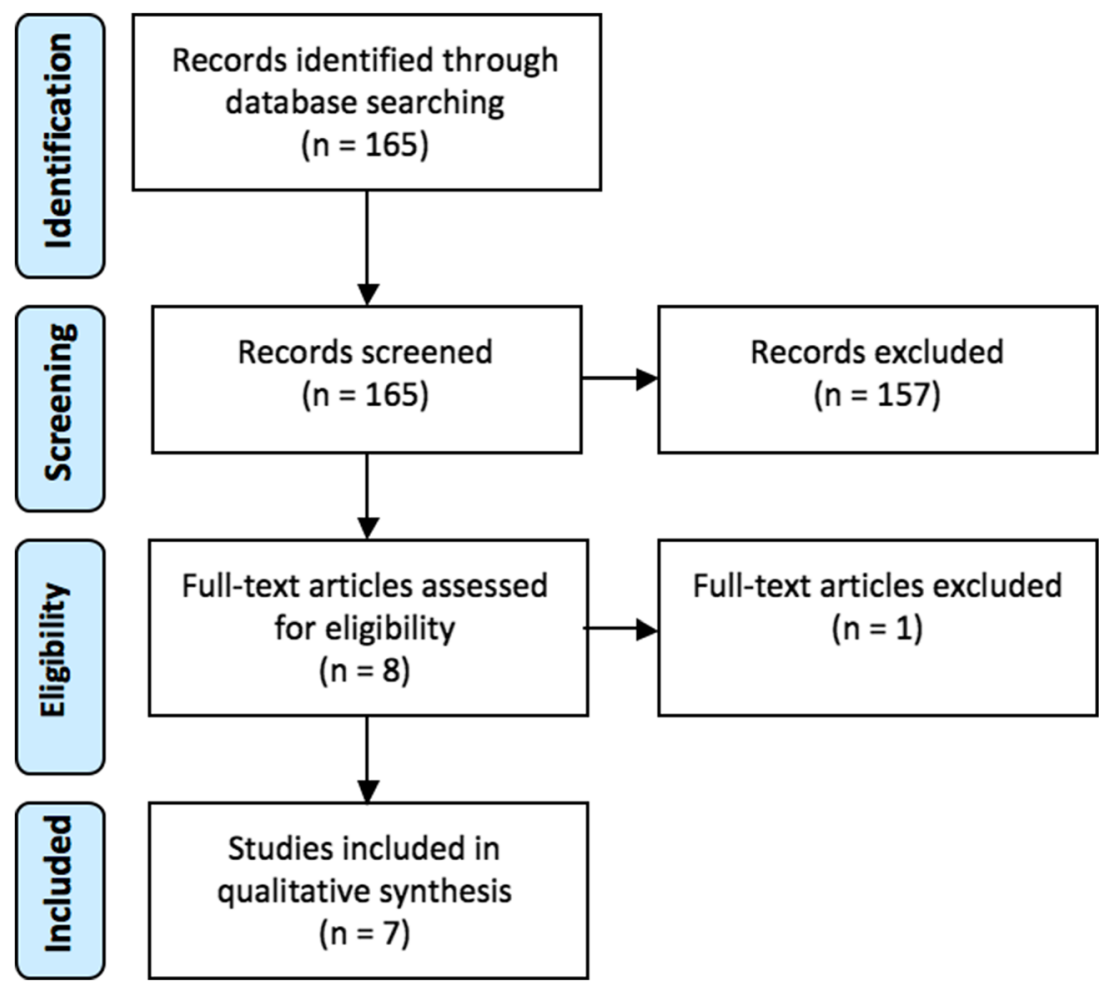

\section{Characteristics of included studies}

Included studies were composed of three congress abstracts [18-20] and four original publications [14-17]. All of them were recent, two being published in 2014 [14, 20], one in 2016 [15], two in 2017 [16, 17], and two in 2018 [18, 19]. All of them were cohort studies [14-20]. Six studies compared perineal wound outcomes between patients with pNPWT and those with conventional wound dressing [14-16, 18-20]. Control patients were historical in 3 studies [14-16] (Table 2).

All studies included patients undergoing APR [14-20] or pelvic exenteration $[14,17,18]$. Surgical indication was mainly rectal cancer [14-17], but two studies also included patients suffering from inflammatory bowel disease [14, 16]. Application of biological mesh to close the perineal defect and/or use of drain was poorly documented and varied among studies (Tables 3 and 4). In patients with application of pNPWT on closed perineum, the device used was the PREVENA incision management system (KCI, Acelity, San Antonio, USA) set at $-100 \mathrm{mmHg}$ [17] or for 5 days [20] and the PICO single use negative pressure wound therapy system (Smith \& Nephew, Hertfordshire, UK) set at $-80 \mathrm{mmHg}$ for 4-8 days [16] or unknown commercial device set at $125 \mathrm{mmHg}$ for 5 days $[14,15]$.

\section{Perineal wound complications}

Wiegering et al. reported one wound dehiscence $(16.7 \%$ of patients) occurring after 8 days and requiring V.A.C. therapy for secondary healing [17].

Chadi et al. compared 27 patients with pNPWT with 32 patients with conventional wound dressing and found pNPWT

Table 2 Characteristics of included studies

\begin{tabular}{llllll}
\hline Authors & Year & Country & Type of publication & Type of study & Period \\
\hline Chadi et al. & 2014 & Canada & Original publication & Retrospective cohort & $2010-2012$ \\
Chung et al. & 2014 & USA & Congress abstract & Retrospective cohort & May 2009-September 2013 \\
Rather et al. & 2018 & USA & Congress abstract & Retrospective cohort & - \\
Sumrien et al. & 2016 & UK & Original publication & Prospective cohort & November 2012-April 2015 \\
Takahashi et al. & 2018 & Japan & Congress abstract & Cohort & - \\
Van der Walk et al. & 2017 & The Netherlands & Original publication & Prospective cohort with historical controls & January 2015-December 2015 \\
Wiegering et al. & 2017 & Germany & Original publication & Cohort & - \\
\hline
\end{tabular}




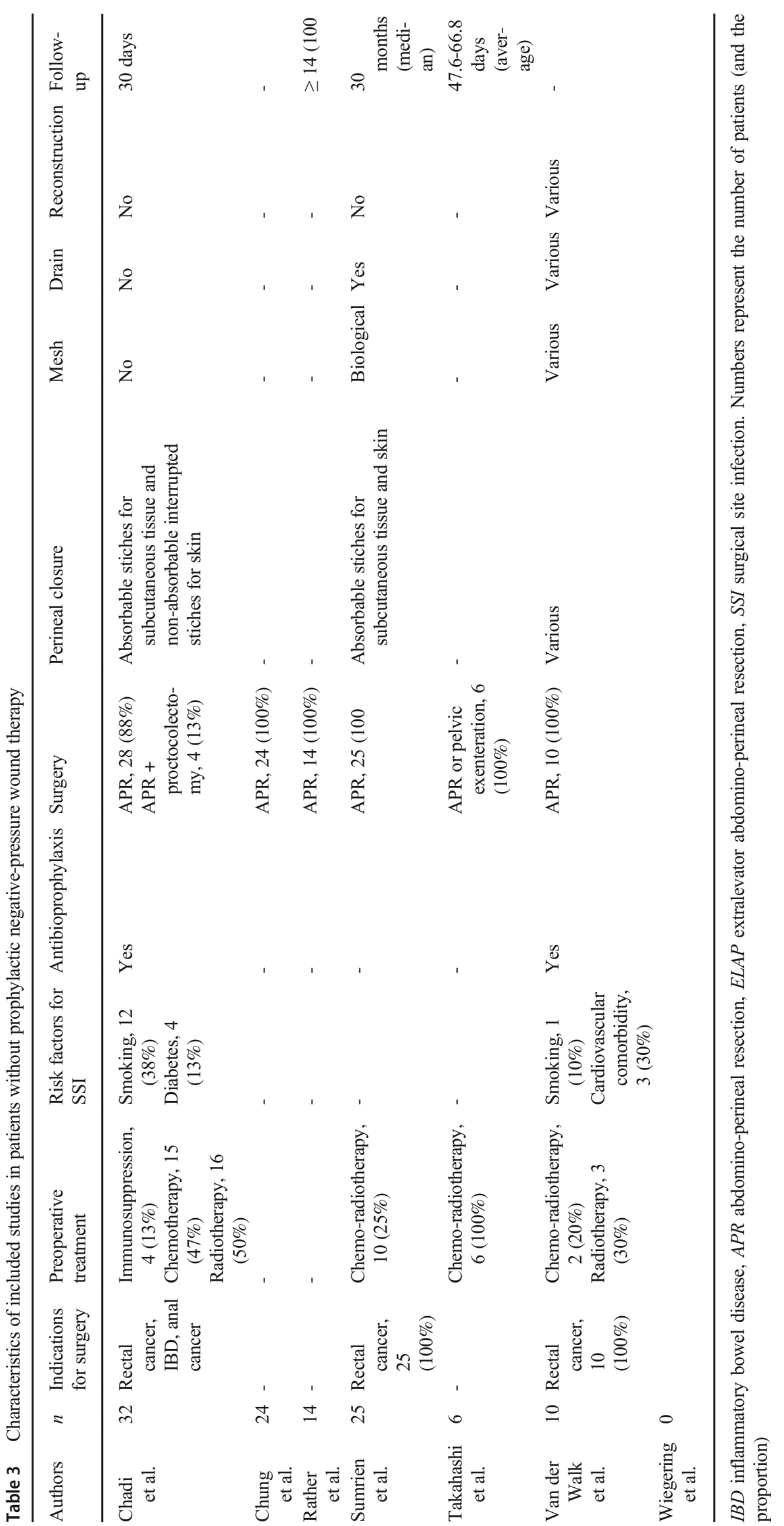




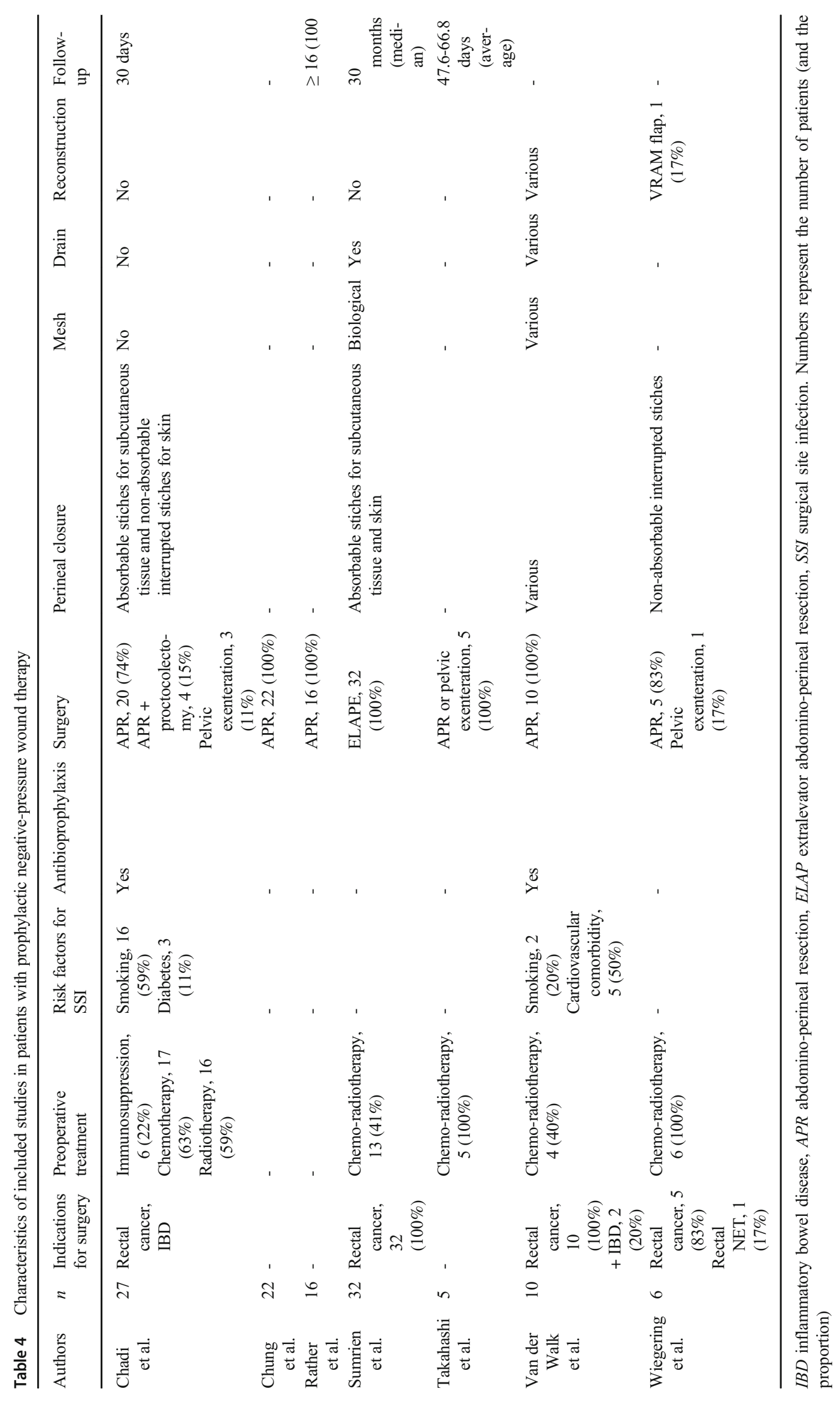




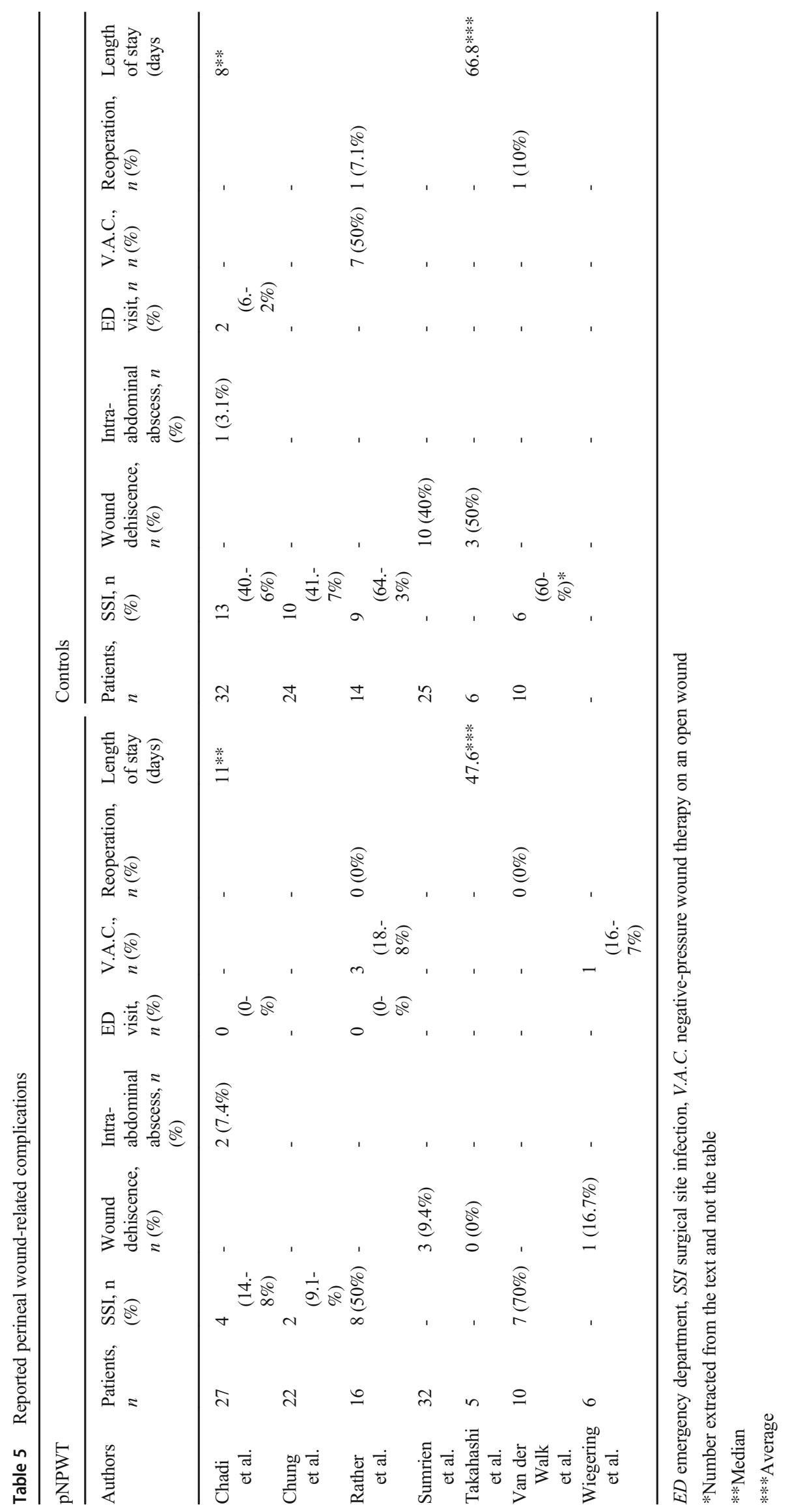


patients to have significantly less SSI than control patients (14.8\% versus $40.6 \%, p=0.04)$. The incidence of intraabdominal abscess $(7.4 \%$ versus $3.1 \%, p=0.59)$ or of emergency department visit $(0 \%$ versus $6.2 \%, p=0.18)$ did not differ between the two groups [14]. Chung et al. also reported significantly lower incidence of SSI among patients with pNPWT than in controls $(9.1 \%$ versus $41.7 \%, p=0.012)$ [20]. Rather et al., however, found similar incidence of SSI between patients with and without pNPWT (50\% versus $64.3 \%$ ) but described these infectious complications to be "less severe" in pNPWT patients. Noteworthy, $18.8 \%$ of pNPWT patients and $50 \%$ of control patients required V.A.C. therapy of the perineal wound for secondary healing. One patient who did not benefited from pNPWT required reoperation [19]. Van der Walk et al. reported similar incidences of SSI between pNPWT and control patients (70\% versus $60 \%, p$ value not communicated). One patient from the conventional wound dressing group required reintervention [16].

Sumrien et al. reported significantly lower incidence of wound dehiscence in pNPWT patients than in controls (40\% versus $9.4 \%, p=0.01)$. Takahashi et al. observed a similar trend ( $0 \%$ versus $50 \%, p$ value not communicated) (Table 5).

\section{Discussion}

In the present systematic review, we have included seven studies investigating the effect of pNPWT on the prevention of perineal wound complications after APR.

Two publications reported significantly lower incidence of SSI in pNPWT patients than in controls with a risk reduction of about 25-30\% [14, 20], therefore showing encouraging results in favor of perineal pNPWT. Two other publications described similar incidence of SSI between the two groups of patients but described SSI in pNPWT patients to be less severe [19], or the authors believed that pNPWT could accelerate wound healing [16]. Further, one study reported significantly lower incidence of wound dehiscence in $\mathrm{pNPWT}$ patients than in controls (40\% versus 9.4\%, $p=0.01$ ) [15].

We note that these studies were pilot studies, which presented several limitations. These were heterogeneous in designs, patients' populations (with potential differences in terms of risk factors for wound-related complications), definition of controls (mainly historical), surgical procedures, pNPWT procedures (in terms of device, negative pressure applied, and duration of therapy - of note, several studies reported dysfunction of the device(s) requiring replacement and/ or discontinuation of therapy $[15,16])$, and reported outcomes (SSI, wound dehiscence, intra-abdominal abscess, emergency department visit, negative-pressure therapy for secondary healing, reoperation) and had small sample sizes, which constitute important limitations to their interpretation and prevent any meta-analysis of the actual literature in the field. However, we note that studies reporting a significant effect of pNPWT on the prevention of SSI and/or wound dehiscence were the studies with the largest sample sizes. Therefore, it might be likely that the absence of a significant effect observed in smaller studies might result from a type II error (lack of statistical power).

We believe that prevention of wound-related complications after APR is of crucial importance. For instance, these complications might increase length of stay with the subsequent risks of thrombo-embolic complications and nosocomial infections in these vulnerable patients and might also postpone administration of adjuvant therapy. We think that perineal application of pNPWT for the prevention of wound-related complications after ARP deserves further investigation, for example, with a large-enough randomized controlled trial, as the existing high incidence of perineal wound complications might allow to obtain important benefits even with a small effect of that therapy.

\section{Conclusion}

The largest non-randomized studies investigating the effect of pNPWT on the prevention of wound-related complications after APR show encouraging results in terms of reduction of SSI and wound dehiscence that deserve further investigation and confirmation.

Funding Open access funding provided by University of Geneva.

\section{Compliance with ethical standards}

Conflict of interest The authors declare that they have no conflict of interest.

Open Access This article is licensed under a Creative Commons Attribution 4.0 International License, which permits use, sharing, adaptation, distribution and reproduction in any medium or format, as long as you give appropriate credit to the original author(s) and the source, provide a link to the Creative Commons licence, and indicate if changes were made. The images or other third party material in this article are included in the article's Creative Commons licence, unless indicated otherwise in a credit line to the material. If material is not included in the article's Creative Commons licence and your intended use is not permitted by statutory regulation or exceeds the permitted use, you will need to obtain permission directly from the copyright holder. To view a copy of this licence, visit http://creativecommons.org/licenses/by/4.0/.

\section{References}

1. Miles WE (1971) A method of performing abdomino-perineal excision for carcinoma of the rectum and of the terminal portion of the pelvic colon (1908). CA Cancer J Clin 21:361-364

2. Heald RJ, Husband EM, Ryall RD (1982) The mesorectum in rectal cancer surgery-the clue to pelvic recurrence? Br J Surg 69:613-616 
3. Holm T, Ljung A, Haggmark $T$ et al (2007) Extended abdominoperineal resection with gluteus maximus flap reconstruction of the pelvic floor for rectal cancer. Br J Surg 94:232-238

4. Schiltz B, Buchs NC, Penna M, Scarpa CR, Liot E, Morel P, Ris F (2017) Biological mesh reconstruction of the pelvic floor following abdominoperineal excision for cancer: a review. World J Clin Oncol 8:249-254

5. Peirce C, Martin S (2016) Management of the perineal defect after abdominoperineal excision. Clin Colon Rectal Surg 29:160-167

6. Sheckter CC, Shakir A, Vo H, Tsai J, Nazerali R, Lee GK (2016) Reconstruction following abdominoperineal resection (APR): indications and complications from a single institution experience. $\mathrm{J}$ Plast Reconstr Aesthet Surg 69:1506-1512

7. Bullard KM, Trudel JL, Baxter NN, Rothenberger DA (2005) Primary perineal wound closure after preoperative radiotherapy and abdominoperineal resection has a high incidence of wound failure. Dis Colon Rectum 48:438-443

8. Artioukh DY, Smith RA, Gokul K (2007) Risk factors for impaired healing of the perineal wound after abdominoperineal resection of rectum for carcinoma. Color Dis 9:362-367

9. Wiatrek RL, Thomas JS, Papaconstantinou HT (2008) Perineal wound complications after abdominoperineal resection. Clin Colon Rectal Surg 21:76-85

10. Webster J, Liu Z, Norman G et al (2019) Negative pressure wound therapy for surgical wounds healing by primary closure. Cochrane Database Syst Rev 3:CD009261

11. Zwanenburg PR, Tol BT, Obdeijn MC et al (2019) Meta-analysis, meta-regression, and GRADE assessment of randomized and nonrandomized studies of incisional negative pressure wound therapy versus control dressings for the prevention of postoperative wound complications. Ann Surg

12. Roos E, Toso C, Meyer J Comment on: Meta-analysis, meta-regression, and GRADE assessment of randomized and nonrandomized studies of incisional negative pressure wound therapy versus control dressings for the prevention of postoperative wound complications. Ann Surg Accepted for publication
13. Moher D, Liberati A, Tetzlaff J, Altman DG, PRISMA Group (2009) Preferred reporting items for systematic reviews and metaanalyses: the PRISMA statement. J Clin Epidemiol 62:1006-1012

14. Chadi SA, Kidane B, Britto K, Brackstone M, Ott MC (2014) Incisional negative pressure wound therapy decreases the frequency of postoperative perineal surgical site infections: a cohort study. Dis Colon Rectum 57:999-1006

15. Sumrien H, Newman P, Burt C, McCarthy K, Dixon A, Pullyblank A, Lyons A (2016) The use of a negative pressure wound management system in perineal wound closure after extralevator abdominoperineal excision (ELAPE) for low rectal cancer. Tech Coloproctol 20:627-631

16. van der Valk MJM, de Graaf EJR, Doornebosch PG, Vermaas M (2017) Incisional negative-pressure wound therapy for perineal wounds after abdominoperineal resection for rectal cancer, a pilot study. Adv Wound Care (New Rochelle) 6:425-429

17. Wiegering A, Dietz UA, Corteville C, Plaßmeier L, Jurowich C, Germer CT, Krajinovic K (2017) Impact of incisional negative pressure wound therapy on perineal wound healing after abdominoperineal rectum extirpation. Int J Color Dis 32:291-293

18. Takahashi Y, Miyoshi N, Nishimura J et al (2018) Negativepressure wound therapy for perineum surgical wound of rectal cancer patients after chemoradiation therapy. Cancer Sci 109

19. Rather A, Fisher A, Nedelcoviciu R et al (2018) Perineal wound complications after initiation of closed incision negative pressure therapy in patients undergoing APR: a comparative study. Dis Colon Rectum 61:5 (e293-e294)

20. Chung A, Vogler S, Finlayson S et al (2014) Incisional negative pressure therapy reduces perineal superficial wound infections following abdominoperineal resection. Dis Colon Rectum 57:5 (e306e307)

Publisher's note Springer Nature remains neutral with regard to jurisdictional claims in published maps and institutional affiliations. 